\title{
Latest tests of hard QCD at HERA
}

\author{
Oleksandr Zenaiev (DESY) \\ on behalf of the $\mathrm{H} 1$ and ZEUS collaborations
}

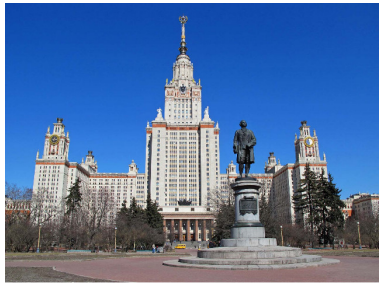

18th Lomonosov Conference, MSU, 23-30 August 2017 


\section{Experimental set-up}

\section{HERA Collider}

- ep collisions

- $\sqrt{\mathrm{s}}=300 \ldots 318 \mathrm{GeV}$ and lower energy runs

\section{$\mathrm{H} 1$ and ZEUS:}

- $4 \pi$ multipurpose detectors

- $\mathcal{L} \sim 500 \mathrm{pb}^{-1}$ per each experiment

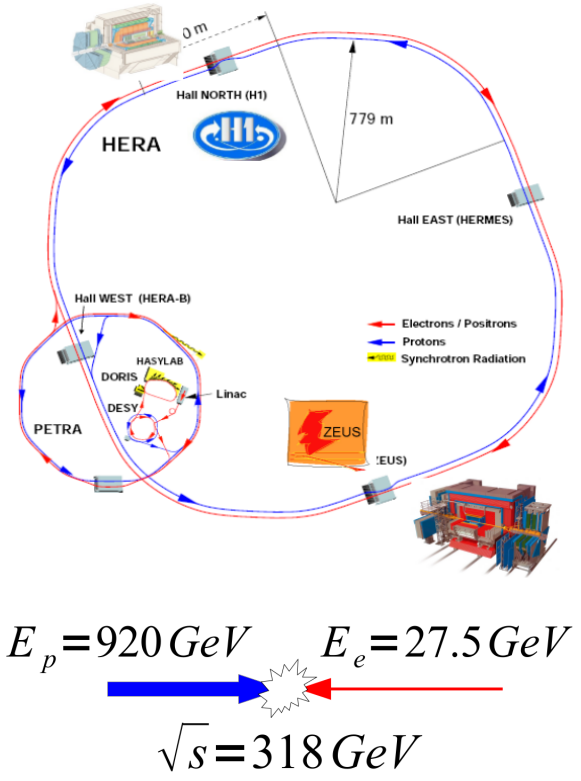




\section{Kinematics}

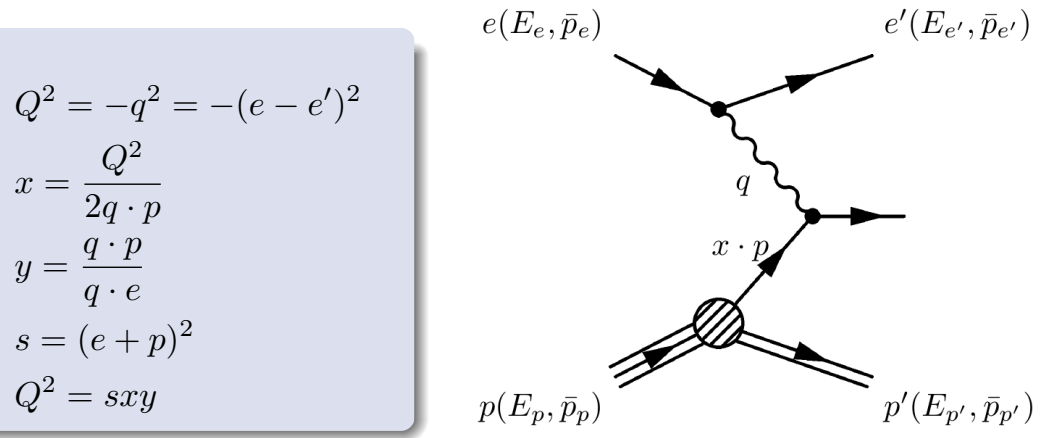

Any two of the variables $\left(Q^{2}, x, y\right)$ define kinematics

$Q^{2}>1 \mathrm{GeV}^{2}-$ deep inelastic scattering (DIS)

$Q^{2}<1 \mathrm{GeV}^{2}-$ photoproduction processes (PHP) 


\section{Overview}

\section{New results from HERA covered in this talk:}

- H1 and ZEUS Collaborations,

"Combination and QCD analysis of beauty and charm production cross section measurements in deep inelastic ep scattering at HERA" [preliminary] H1prelim-17-071, ZEUS-prel-17-01

https://www.desy.de/h1zeus/combined_results/index.php?do=heavy_flavours

- H1 Collaboration, "Measurement of Jet Production Cross Sections in Deep-inelastic ep Scattering at HERA" EPJ C77 (2017) 215, EPJ C75 (2015) 65, H1prelim-17-031

- ZEUS Collaboration, "Further measurements of isolated photons accompanied by jets in deep inelastic ep scattering" [preliminary] ZEUS-prel-16-001 


\section{Overview}

\section{New results from HERA covered in this talk:}

- H1 and ZEUS Collaborations,

"Combination and QCD analysis of beauty and charm production cross section measurements in deep inelastic ep scattering at HERA" [preliminary] H1prelim-17-071, ZEUS-prel-17-01

https://www.desy.de/h1zeus/combined_results/index.php?do=heavy_flavours

- H1 Collaboration,

"Measurement of Jet Production Cross Sections in Deep-inelastic ep Scattering at HERA"

EPJ C77 (2017) 215, EPJ C75 (2015) 65, H1prelim-17-031

- ZEUS Collaboration, "Further measurements of isolated photons accompanied by jets in deep inelastic ep scattering" [preliminary] ZEUS-prel-16-001 


\section{Heavy flavour (HF) production in DIS}

Test of pQCD (multiple hard scales: $\left.Q^{2}, p_{T}(Q), m_{Q}\right)$

Charm and beauty in DIS are predominantly produced via Boson-Gluon Fusion (BGF) process

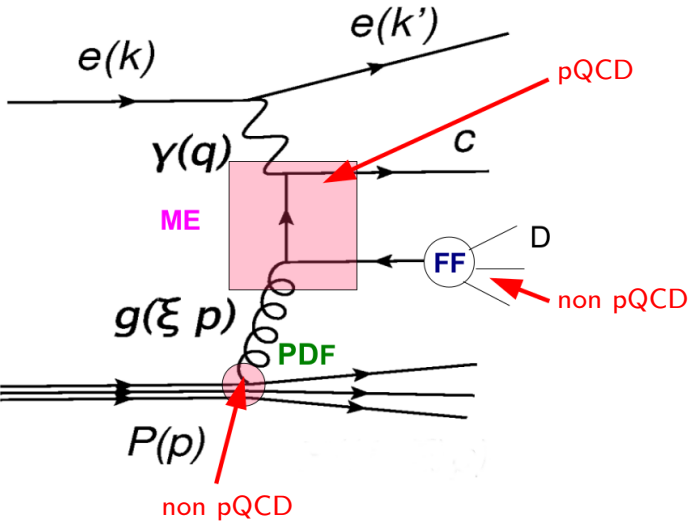

Production is directly sensitive to $g$ PDF in the proton and to HQ masses

PDF: parton distribution functions

ME: (hard) matrix element

FF: fragmentation function \& fraction 


\section{pQCD approximation of heavy flavour production}

Fixed Flavour Number Scheme (FFNS)

- c,b-quarks are massive $\Rightarrow$ not a part of the proton, produced perturbatively in hard scattering

- valid for $Q^{2} \sim m_{c, b}^{2}$

Zero Mass Variable Flavour Number Scheme (ZMVFNS)

- c,b-quarks are massless $\Rightarrow$ a part of the proton

- valid for $Q^{2}>>m_{c, b}^{2}$

General Mass Variable Flavour Number Scheme (GMVFNS)

- equivalent to FFNS at low $Q^{2}$

- equivalent to ZMVFNS at high $Q^{2}$

- not unique (RT, ACOT, ...)

detailed discussion in [EPJ C73 (2013) 2311] 


\section{Input data}

\begin{tabular}{|c|c|c|c|c|c|c|c|}
\hline Data set & Tagging & & $\begin{array}{l}2 \text { range } \\
\left.\mathrm{GeV}^{2}\right]\end{array}$ & $N_{c}$ & $\begin{array}{r}\mathscr{L} \\
{\left[\mathrm{pb}^{-1}\right]}\end{array}$ & $\begin{array}{r}\sqrt{s} \\
{[\mathrm{GeV}]}\end{array}$ & $N_{b}$ \\
\hline 1 H1 VTX [8] & VTX & 5 & 2000 & 29 & 245 & 318 & \\
\hline $2 \mathrm{H} 1 D^{*+}$ HERA-I [9] & $D^{*+}$ & 2 & 100 & 17 & 47 & 318 & \\
\hline 3 H1 $D^{*+}$ HERA-II (medium $Q^{2}$ ) [10] & $D^{*+}$ & 5 & 100 & 25 & 348 & 318 & \\
\hline 4 H1 $D^{*+}$ HERA-II (high $Q^{2}$ ) [11] & $D^{*+}$ & 100 & -1000 & 6 & 351 & 318 & \\
\hline 5 ZEUS $D^{*+} 96-97[12]$ & $D^{*+}$ & 1 & 200 & 21 & 37 & 300 & \\
\hline 6 ZEUS $D^{*+} 98-00$ [13] & $D^{*+}$ & 1.5 & 1000 & 31 & 82 & 318 & \\
\hline 7 ZEUS $D^{0} 2005$ [14] & $D^{0}$ & 5 & 1000 & 9 & 134 & 318 & \\
\hline 8 ZEUS $\mu$ 2005 [7] & $\mu$ & 20 & -10000 & 8 & 126 & 318 & \\
\hline 9 ZEUS $D^{+}$HERA-II [2] & $D^{+}$ & 5 & 1000 & 14 & 354 & 318 & \\
\hline 10 ZEUS $D^{*+}$ HERA-II [3] & $D^{*+}$ & 5 & 1000 & 31 & 363 & 318 & \\
\hline 11 ZEUS VTX HERA-II [4] & VTX & 5 & 1000 & 18 & 354 & 318 & 17 \\
\hline 12 ZEUS $e$ HERA-II [5] & $e$ & 10 & 1000 & & 363 & 318 & \\
\hline 13 ZEUS $\mu+$ jet HERA-I [6] & $\mu$ & 2 & 3000 & & 114 & 318 & 11 \\
\hline
\end{tabular}

(corresponding references can be found in backup)

- Combined reduced cross sections: $\sigma_{\text {red }}^{Q \bar{Q}}=\frac{\mathrm{d}^{2} \sigma^{Q \bar{Q}}}{\mathrm{~d} x_{\mathrm{Bj}} \mathrm{d} Q^{2}} \cdot \frac{x_{\mathrm{Bj}} Q^{4}}{2 \pi \alpha^{2}\left(1+(1-y)^{2}\right)}$

- Combined data provided in kinematic range: $2.5 \leq Q^{2} \leq 2000 \mathrm{GeV}^{2}, 3 \times 10^{-5} \leq x_{\mathrm{Bj}} \leq 5 \times 10^{-2}$

- Input $209 c, 52 b$ data points $\Rightarrow$ combined $52 c, 27 b$ points

- Extends previous HERA charm combination with 3 new $c$ data sets and 5 new $b$ : first combination of HERA $b$ data 


\section{Combined data}

- H1 VTX

ZEUS $\mu 2005$

- ZEUS D ${ }^{0}$

म ZEUS VTX
CHARM

H1 D* HERA-II $>$ H1 D* HERA-I ZEUS D* 98-00 $\triangle$ ZEUS D* 96-97

क ZEUS D

* ZEUS D* HERA-II

- HERA (prel.)
H1 and ZEUS

preliminary

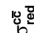

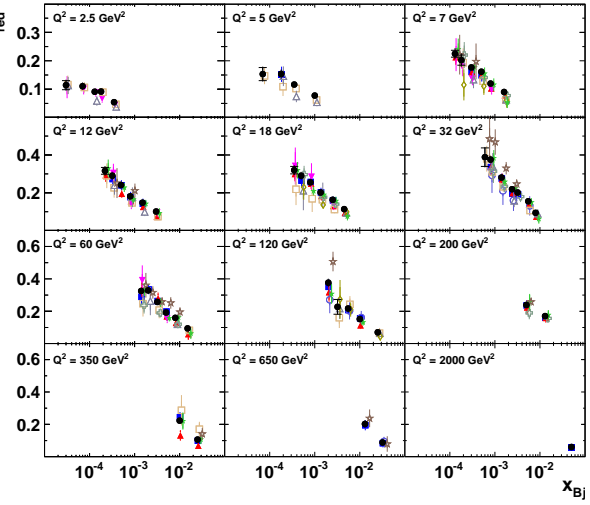

$\chi^{2} /$ dof $=149 / 187$

$\rightarrow$ input data are consistent

$\rightarrow$ significantly reduced uncertainties as compared to the individual measurements

BEAUTY
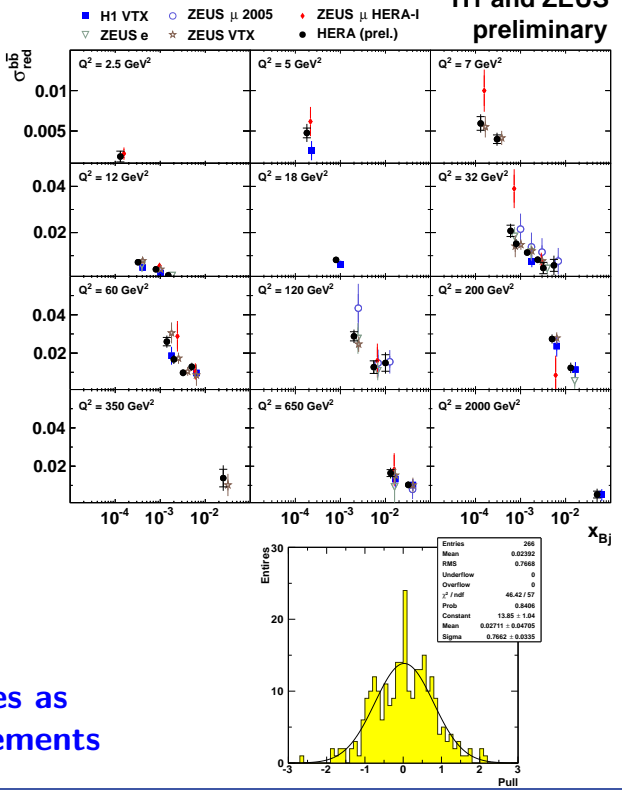


\section{BACKUP. Combined data}

\section{CHARM}

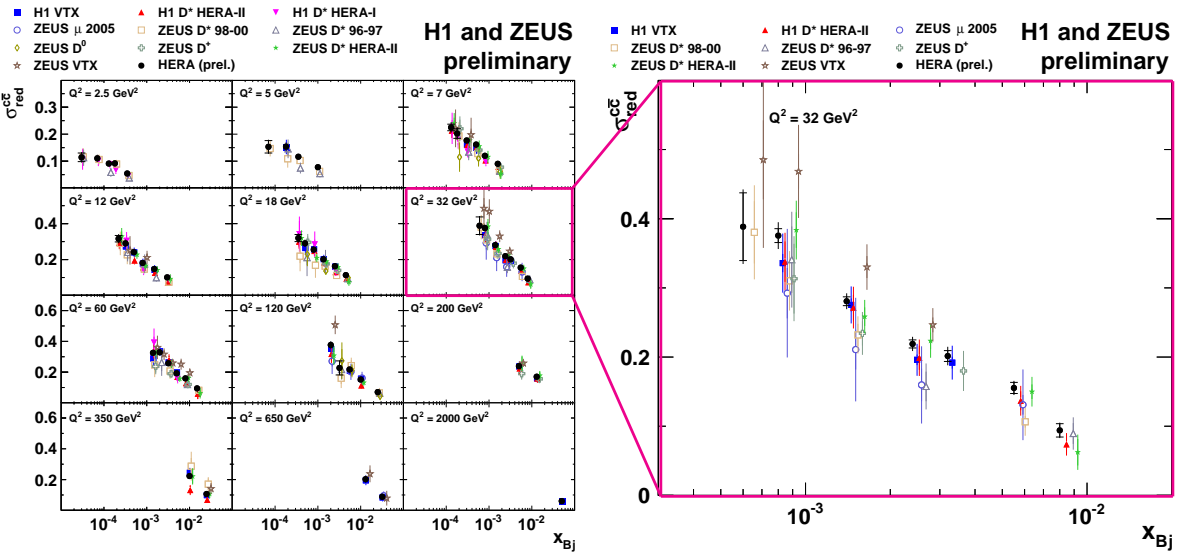

Significantly improved precision compared to input measurements 


\section{Theoretical predictions compared to combined data}

\section{Predictions calculated with OPENQCDRAD interfaced in xFitter}

www-zeuthen.desy.de/ alekhin/OPENQCDRAD

ww. xfitter.org

- input PDFs: HERAPDF2.0FF3A, ABM11, ABMP16, or fitted

- NLO or approx. NNLO as implemented in OPENQCDRAD

- $\mu_{f}=\mu_{r}=\sqrt{Q^{2}+4 m_{Q}^{2}}$, varied by factor 2 (dominant unc.)

- $m_{c}\left(m_{c}\right)=1.27 \pm 0.03 \mathrm{GeV}$, $m_{b}\left(m_{b}\right)=4.18 \pm 0.03 \mathrm{GeV}$ [PDG2016], or fitted

FFN scheme, $n_{f}=3$ : reliable in this kinematic range

\section{CHARM (beauty in BACKUP)}

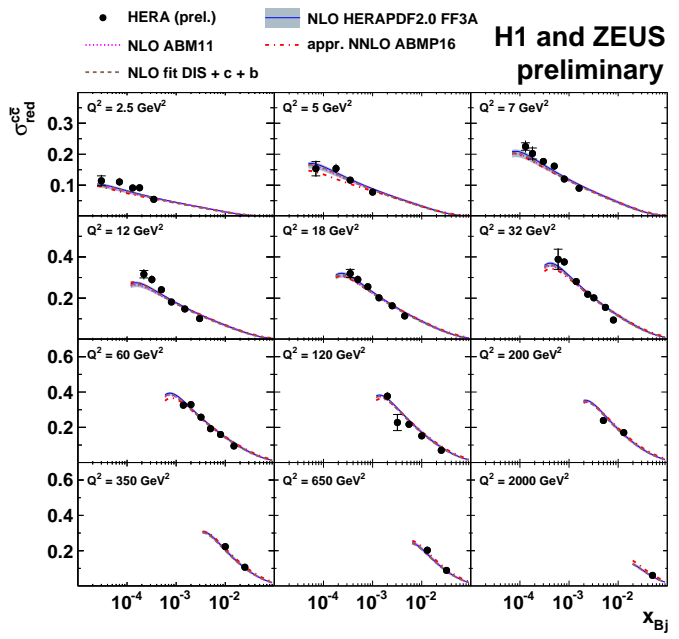

Overall reasonable description, some $x$ slope at low and medium $Q^{2}$ 


\section{Theoretical predictions compared to combined data}

Predictions calculated with OPENQCDRAD interfaced in xFitter

www-zeuthen.desy.de/ alekhin/OPENQCDRAD ww. xfitter.org

- input PDFs: HERAPDF2.0FF3A, ABM11, ABMP16, or fitted

- NLO or approx. NNLO as implemented in OPENQCDRAD

- $\mu_{f}=\mu_{r}=\sqrt{Q^{2}+4 m_{Q}^{2}}$, varied by factor 2 (dominant unc.)

- $m_{c}\left(m_{c}\right)=1.27 \pm 0.03 \mathrm{GeV}$, $m_{b}\left(m_{b}\right)=4.18 \pm 0.03 \mathrm{GeV}$ [PDG2016], or fitted

FFN scheme, $n_{f}=3$ : reliable in this kinematic range

\section{CHARM (beautv in BACKUP)}

- HERA (prel.) NLO HERAPDF2.0 FF3A NLO ABM11 -.... appr. NNLO ABMP16 H1 and ZEUS NLO fit DIS + c + b preliminary

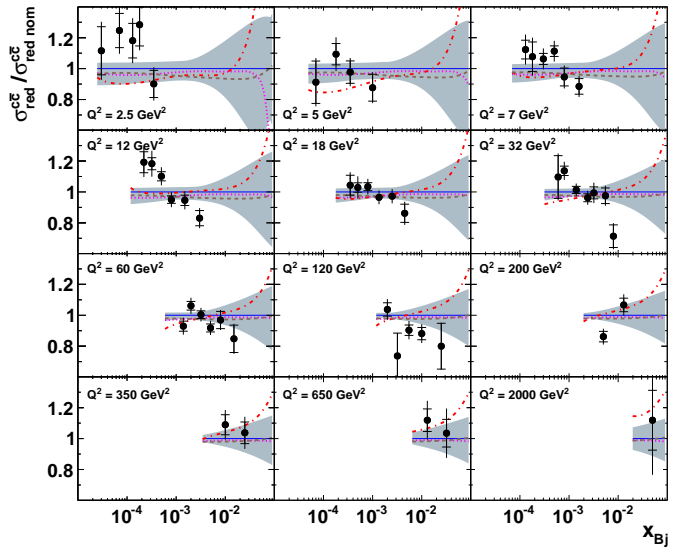

Overall reasonable description, some $x$ slope at low and medium $Q^{2}$ Small sensitivity to PDFs, appr. NNLO do not improve description 


\section{QCD analysis of combined charm and beauty data}

\section{Similar to HERAPDF2.0 FF:}

- performed using xFitter [www.xfitter.org]

- inclusive HERA data + new combined $c \& b$ data

- NLO DGLAP [QCDNUM] and matrix elements [OPENQCDRAD], $n_{f}=3$

- $\mu_{f}=\mu_{r}=\sqrt{Q^{2}+4 m_{Q}^{2}}$ varied by factor 2 (model unc.)

- free $m_{c}\left(m_{c}\right), m_{b}\left(m_{b}\right)$

- $\alpha_{s}\left(M_{Z}\right)^{n_{f}=3}=0.106\left(\rightarrow \alpha_{s}\left(M_{Z}\right)^{n_{f}=5}=0.118\right)$

- HERAPDF parametrisation, $14 \mathrm{p}$

- fit uncertainty using $\Delta \chi^{2}=1$

- model and parametrisation uncertainties

$m_{c}\left(m_{c}\right)=1290_{-41}^{+46}(\mathrm{fit})_{-14}^{+62}(\bmod )_{-31}^{+7}($ par $) \mathrm{MeV}$

$m_{b}\left(m_{b}\right)=4049_{-109}^{+104}(\mathrm{fit})_{-32}^{+90}(\bmod )_{-31}^{+1}(\mathrm{par}) \mathrm{MeV}$

$\Rightarrow$ determined precise HQ masses consistent with world average

PDG2016: $m_{c}\left(m_{c}\right)=1270 \pm 30 \mathrm{MeV}, m_{b}\left(m_{b}\right)=4180_{-30}^{+40} \mathrm{MeV}$ 


\section{Running of $m_{c}$ from HERA DIS data}

Gizsko et al., arXiv:1705.08863 (work partially done within scope of PROSA, ZEUS and H1 collaborations)

18

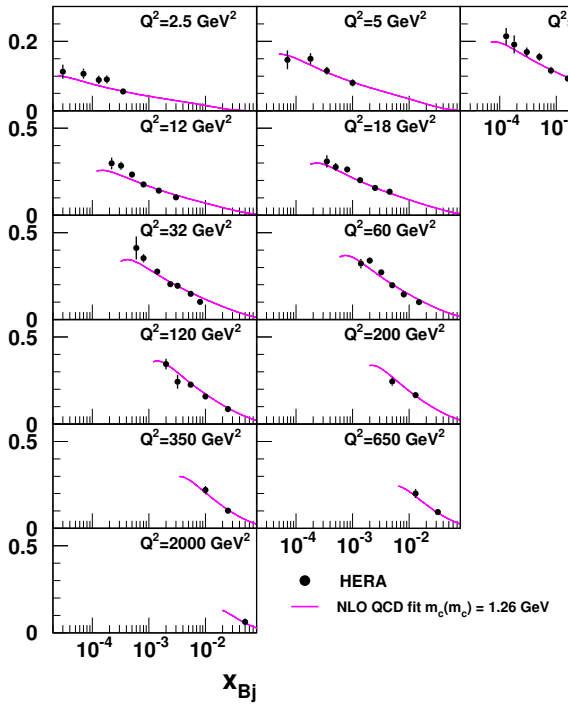

$Q^{2}=7 \mathrm{GeV}^{2}$

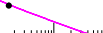

$\mathbf{X}_{\mathrm{Bj}}$
- Determined using earlier published HERA charm data [EPJ C73 (2013) 2311]

- $\overline{\mathrm{MS}}$ charm mass $m_{c}\left(m_{c}\right)$ extracted in regions of $Q^{2}$ and translated to appropriate scale $\mu$<smiles>[AlH2]</smiles>

check of QCD running mass concept

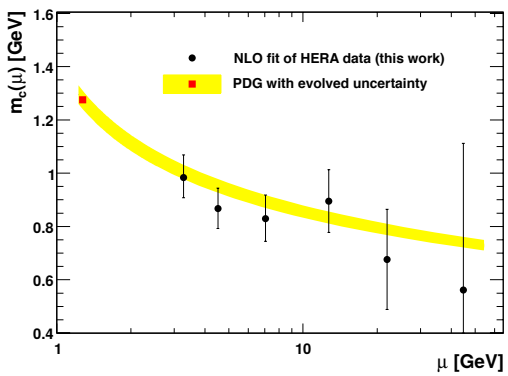




\section{Overview}

\section{New results from HERA covered in this talk:}

- H1 and ZEUS Collaborations, "Combination and QCD analysis of beauty and charm production cross section measurements in deep inelastic ep scattering at HERA" [preliminary] H1prelim-17-071, ZEUS-prel-17-01 https://www.desy.de/h1zeus/combined_results/index.php?do=heavy_flavours

- H1 Collaboration, "Measurement of Jet Production Cross Sections in Deep-inelastic ep Scattering at HERA" EPJ C77 (2017) 215, EPJ C75 (2015) 65, H1prelim-17-031

- ZEUS Collaboration, "Further measurements of isolated photons accompanied by jets in deep inelastic ep scattering" [preliminary] ZEUS-prel-16-001 


\section{H1 multijet production in NC DIS}

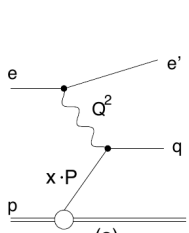

(a)

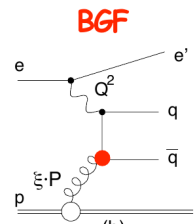

(b)

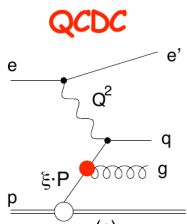

(c)

elweak coupling

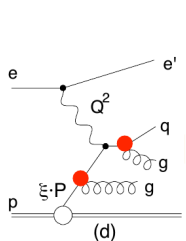

$\propto \alpha_{S}^{2}$

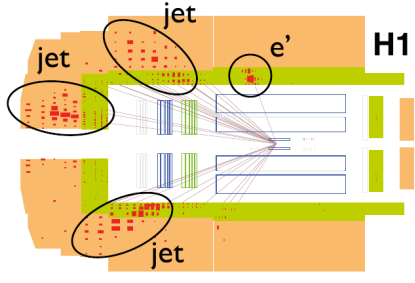

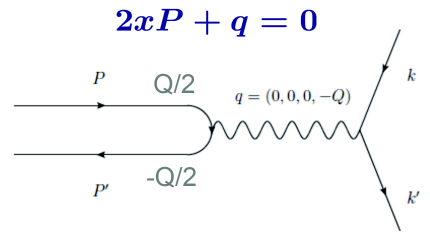

- Breit frame: separates QCD processes from EW

- QCD jets at $L O$ are produced via Boson-Gluon Fusion (BGF) and QCD Compton (QCDC):

$\rightarrow$ probe $g \cdot \alpha_{S}$ at $L O$

- Jets are reconstructed using $k_{T}$ algorithm

- Phase space:

DIS:

$$
5.5<Q^{2}<80 \mathrm{GeV}^{2}
$$$$
0.2<y<0.6
$$

inclusive jets

$$
4.5<p_{T}^{\text {jet }}<50 \mathrm{GeV}
$$$$
-1.0<\eta^{\text {lab }}<2.5
$$

dijets

trijets

$$
5.0<\left\langle p_{T}^{\text {jet }}\right\rangle_{2}<50 \mathrm{GeV}
$$$$
5.5<\left\langle p_{T}^{\text {jet }}\right\rangle_{3}<40 \mathrm{GeV}
$$

+ extension of high- $Q^{2}$ meas. [EPJ C75 (2015) 65]

- Simultaneous unfolding of (multi)jet and NC DIS events, respecting all statistical correlations 


\section{H1 multijets normalised to NC DIS}

\section{Dijets / NC DIS / NLO}
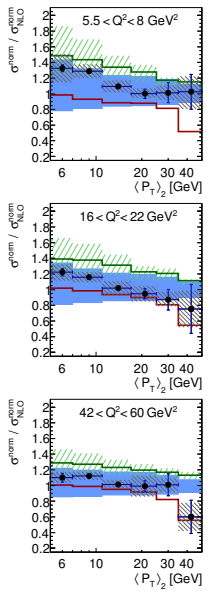
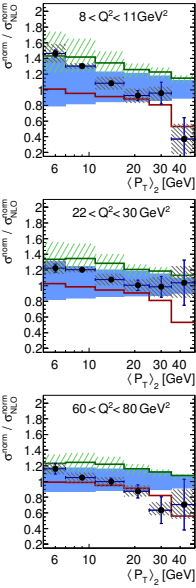
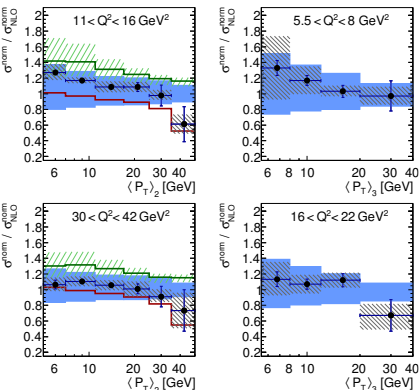

H1 Normalised dijets

\ H1 HERA-II

Systematic uncertainty

NLO $\otimes$ hadr. corr.

— aNNLO $\otimes$ hadr. corr.

$\Longrightarrow$ NNLO $\otimes$ hadr. corr.

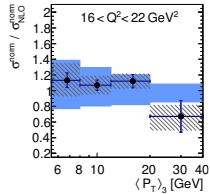

Trijets / NC DIS / NLO

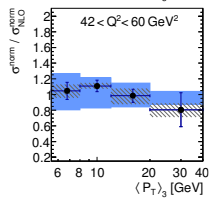

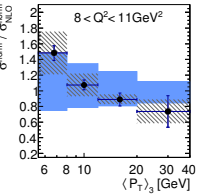
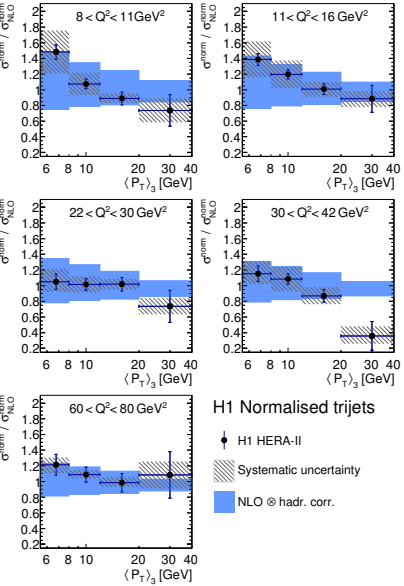

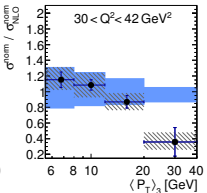

$\mathrm{H} 1$ Normalised trijets

\. H1 HERA-I

Systematic uncertainty

NLO $\otimes$ hadr. corr.

- Experimental systematic unc. partially cancel in ratio Jets / NC DIS - Improved description of dijets shape by NNLO calculations 


\section{H1 multijets: extraction and running of $\boldsymbol{\alpha}_{S}$ at NNLO}

H1 Collaboration and V. Bertone, J. Currie, T. Gehrmann, C. Gwenlan, A. Huss, J. Niehues, M. Sutton

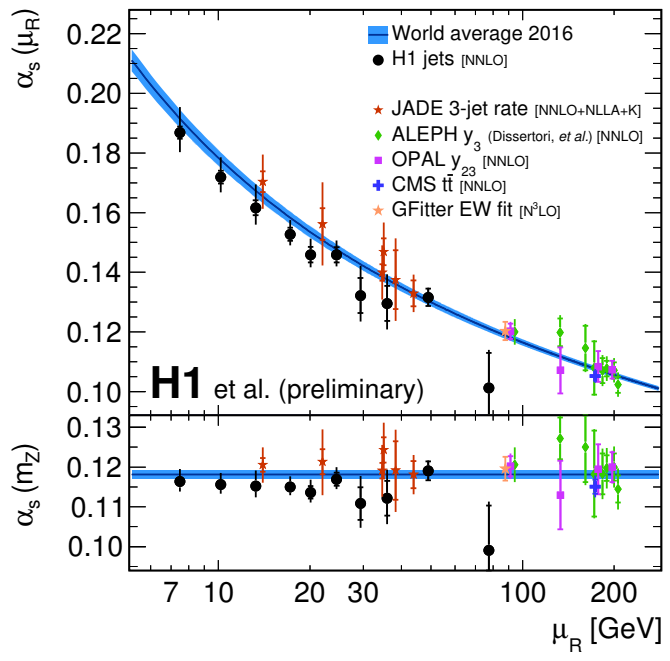

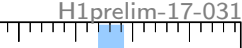

H1 et al. (preliminary)

H1 inclusive jets [all NNLO] $300 \mathrm{GeV}$ high- $\mathrm{Q}^{2}$ HERA-I low-Q ${ }^{2}$ HERA-I high-Q ${ }^{2}$ HERA-II low-Q ${ }^{2}$ HERA-II high-Q ${ }^{2}$

H1 dijets [all NNLO] $300 \mathrm{GeV}$ high- $\mathrm{Q}^{2}$ HERA-I IOW-Q ${ }^{2}$ HERA-II low-Q ${ }^{2}$ HERA-II high-Q ${ }^{2}$

H1 inclusive jets [NNLO] $\mathrm{H} 1$ dijets [NNLO]

H1 jets [NNLO]

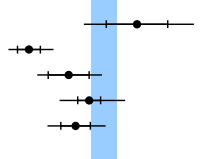

World average [2016]

Fit to inclusive and dijet data: $\chi^{2} / n_{\text {dof }}=1.03, n_{\text {dof }}=203$ $\alpha_{S}\left(m_{Z}\right)=0.1157(6)_{\exp }(3)_{\mathrm{had}}(6)_{\mathrm{PDF}}(12)_{\mathrm{PDF} \alpha_{S}}(2)_{\mathrm{PDFset}}\left(\begin{array}{l}+27 \\ -21\end{array}\right)$ scale 


\section{Overview}

\section{New results from HERA covered in this talk:}

- H1 and ZEUS Collaborations,

"Combination and QCD analysis of beauty and charm production cross section measurements in deep inelastic ep scattering at HERA" [preliminary] H1prelim-17-071, ZEUS-prel-17-01

https://www.desy.de/h1zeus/combined_results/index.php?do=heavy_flavours

- H1 Collaboration,

"Measurement of Jet Production Cross Sections in Deep-inelastic ep Scattering at HERA"

EPJ C77 (2017) 215, EPJ C75 (2015) 65, H1prelim-17-031

-

ZEUS Collaboration, "Further measurements of isolated photons accompanied by jets in deep inelastic ep scattering" [preliminary] ZEUS-prel-16-001 


\section{ZEUS isolated photons in DIS}

ZEUS-prel-16-001, complements ZEUS publication PLB 715 (2012) 88: new variables

QQ

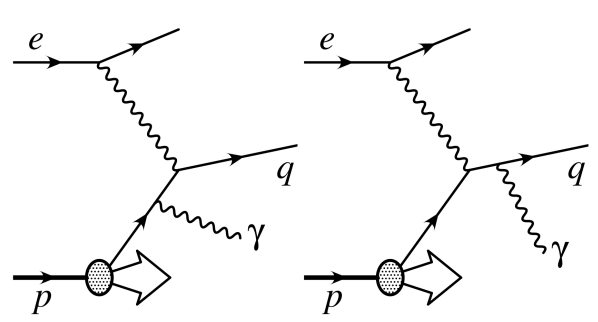

Isolated (prompt) photons can be radiated from:

- quarks $(\mathrm{QQ})$ : part of hard process, provide insights into QCD

- leptons (LL)

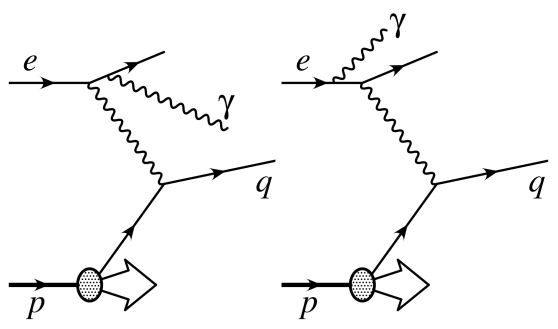

Phase space:

DIS $10<Q^{2}<350 \mathrm{GeV}^{2}$

$\begin{array}{ll}\gamma \quad & 4<E_{T}^{\gamma}<15 \mathrm{GeV} \\ & -0.7<\eta^{\gamma}<0.9\end{array}$

jet $\quad 2.5<E_{T}^{\text {jet }}<35 \mathrm{GeV}$

$-1.5<\eta^{\text {jet }}<1.8$ 


\section{ZEUS isolated photons in DIS: signal/background separation}

\section{ZEUS preliminary}

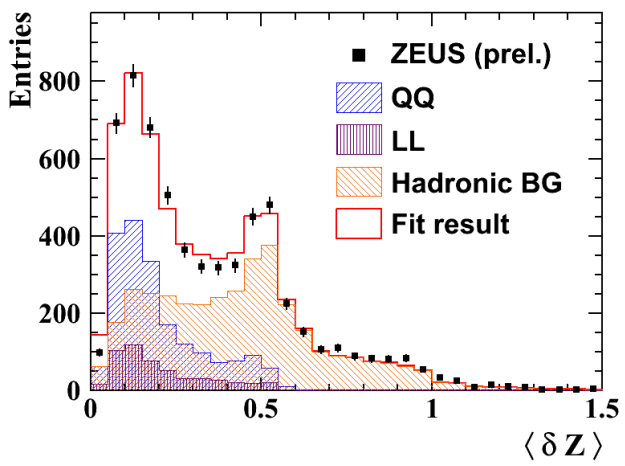

$\langle\delta Z\rangle=\Sigma_{i} E_{i}\left|Z_{i}-Z_{\text {cluster }}\right| /\left(w_{\text {cell }} \Sigma_{i} E_{i}\right)$

$\delta Z$ is energy-weighted mean width of the electromagnetic shower (cluster) in calorimeter relative to its centroid
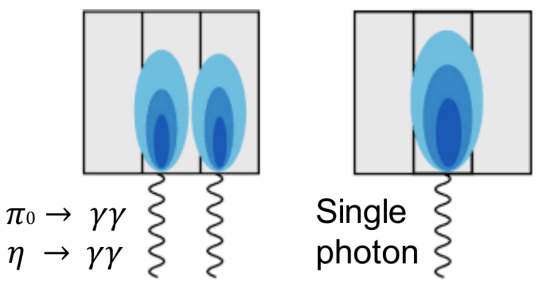

$\Rightarrow$ calorimeter granularity was used to separate prompt $\gamma(\mathrm{QQ}$ and $\mathrm{LL}$ ) from hadronic background (e.g. $\pi^{0} \rightarrow \gamma \gamma$ ) 


\section{ZEUS isolated photons in DIS: comparison to MC models}

\section{ZEUS preliminary}
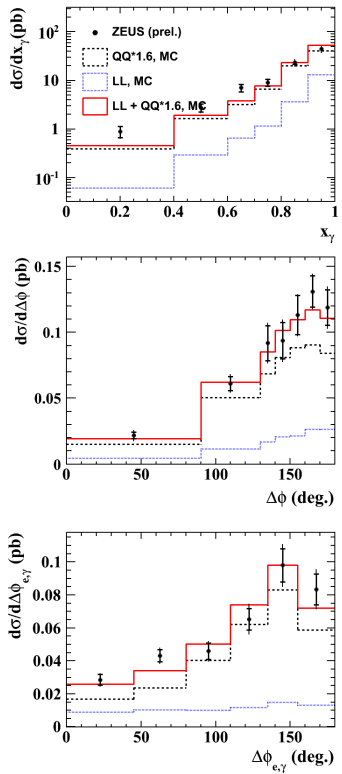
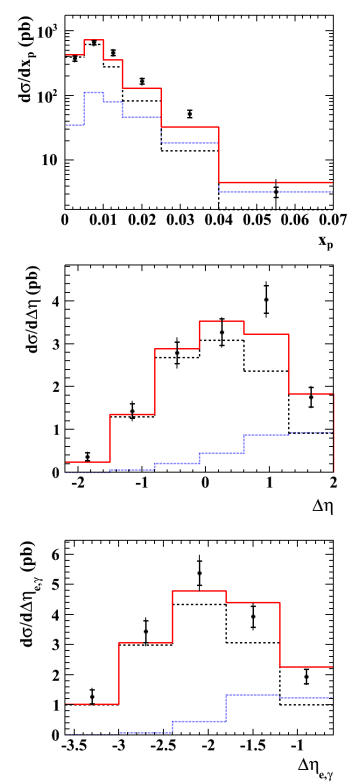

Djangoh (LL) + Pythia (QQ):

- QQ contrbution scaled by 1.6

- LL contribution taken as predicted

$\rightarrow$ good description for all variables
- $x_{\gamma}=\frac{E^{\gamma}-p_{z}^{\gamma}+E^{\mathrm{jet}}-p_{z}^{\mathrm{jet}}}{2 E_{e} y_{J B}}$
- $x_{p}=\frac{E^{\gamma}+p_{z}^{\gamma}+E^{\mathrm{jet}}+p_{z}^{\mathrm{jet}}}{2 E_{p}}$
- $\Delta \phi=\phi^{\text {jet }}-\phi^{\gamma}$
- $\Delta \eta=\eta^{\text {jet }}-\eta^{\gamma}$
- $\Delta \phi_{e, \gamma}=\phi^{e}-\phi^{\gamma}$
- $\Delta \eta_{e, \gamma}=\eta^{e}-\eta^{\gamma}$ 


\section{ZEUS isolated photons in DIS: comparison to QCD predict.}

\section{ZEUS Preliminary 16-001}
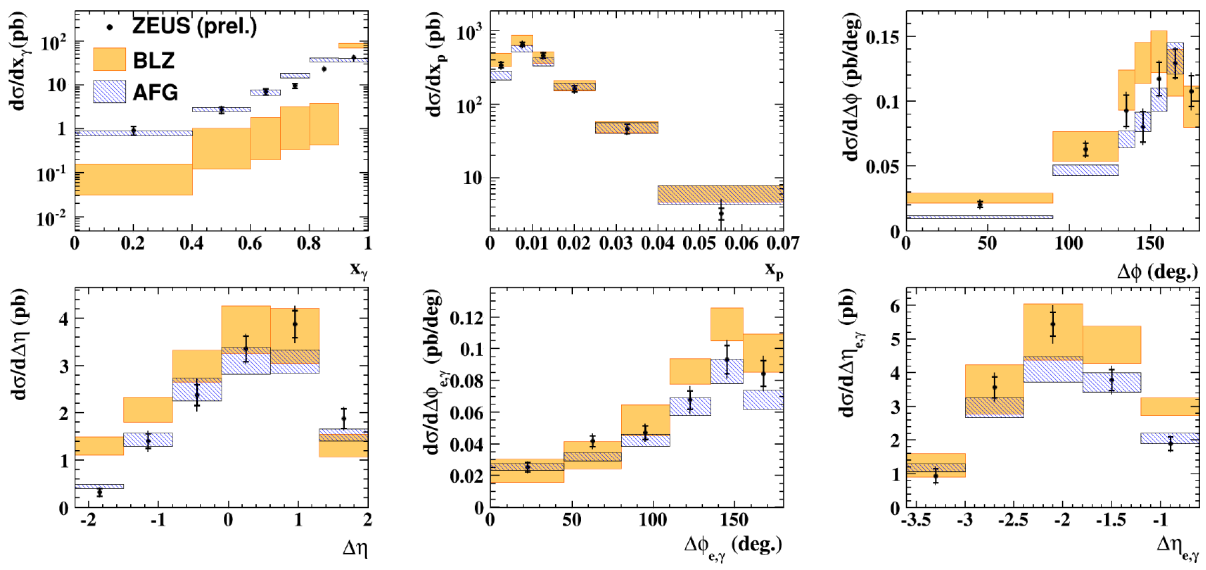

- NLO collinear factorisation by Aurenche, Fontannaz and Guillet (AFG) [1704.08074] $\rightarrow$ describe all variables well

- $k_{T}$-factorisation by Baranov, Lipatov and Zotov (BLZ) [PRD81 (2010) 094034] $\rightarrow$ fair agreement, except $x_{\gamma}$ 


\section{Summary}

New preliminary combined HERA HQ data:

- improvement in precision w.r.t previous HERA results on charm

- first combined HERA results on beauty

- enables precise determination of charm and beauty masses

[H1prelim-17-071, ZEUS-prel-17-01]

https://www.desy.de/h1zeus/combined_results/index.php?do=heavy_flavours

Measurement of multijet production by $\mathrm{H} 1$ :

- high precision data over wide $Q^{2}, p_{T}$ kinematic range

- successfull test of recently appeared NNLO calculations: improved $p_{T}$ shape at NNLO, smaller scale uncertainties

- enables precise determination and check of running of $\alpha_{S}$

[EPJ C77 (2017) 215, EPJ C75 (2015) 65, H1prelim-17-031]

\section{Measurement of isolated photons by ZEUS:}

- good agreement with NLO collinear factorisation predictions

- worser agreement with $k_{T}$-factorisation predictions

[ZEUS-prel-16-001]

$H 1$ and ZEUS continue producing valuable QCD results after 10 years of HERA shutdown! 


\section{BACKUP}




\section{BACKUP. Measurement of charm production at HERA}

\section{"Golden" decay channel $D^{*} \rightarrow D^{0}(K \pi) \pi_{s}$}

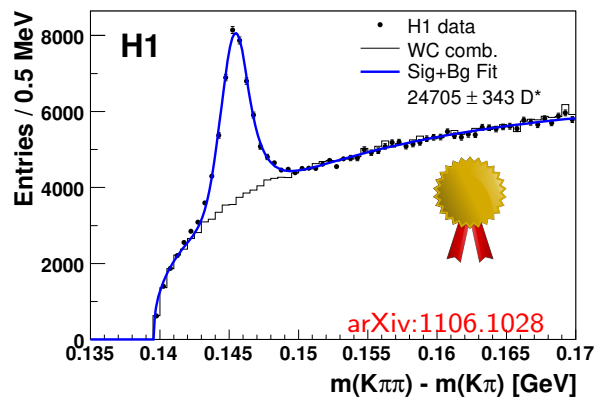

Dedicated H1ZEUS combination:

"Combination of differential $D^{* \pm}$ cross-section measurements in deep-inelastic ep scattering at HERA" [JHEP09 (2015) 149]
Weakly decaying charm hadrons ZEUS
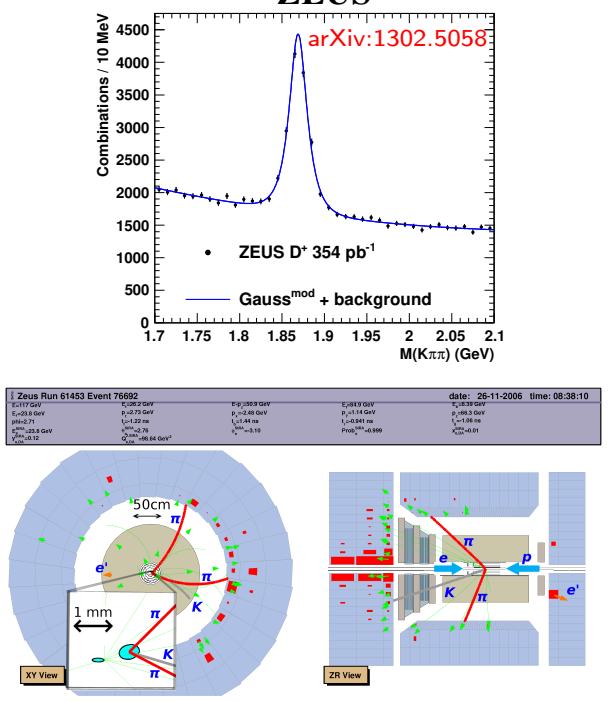


\section{BACKUP. Measurement of $c$ and $b$ production at HERA}

\section{Semi-leptonic (SL) HQ decays}

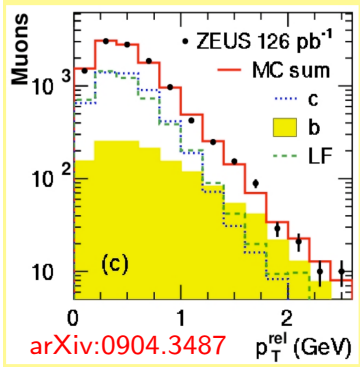

\section{Inclusive lifetime tagging}

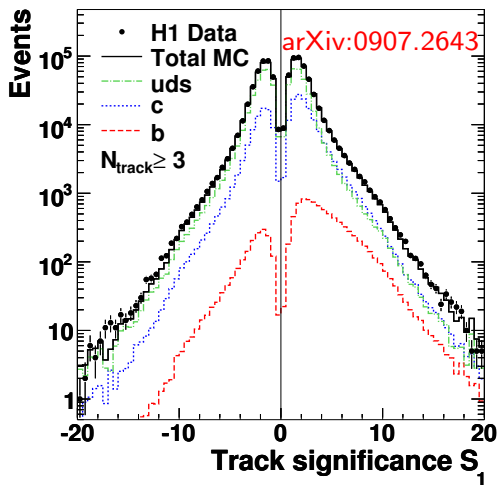

Recent reviews of HF production at HERA:

- O. Behnke, A. Geiser, M. Lisovyi, "Charm, Beauty and Top at HERA", Prog. Part. Nucl. Phys. 84 (2015) 1

- O.Z., "Charm Production and QCD Analysis at HERA and LHC ", Eur. Phys. J. C77 (2017) 151 


\section{BACKUP. Combination procedure}

- fiducial cross sections extrapolated to full phase space using consistent NLO predictions [HVQDIS], account for relevant unc.

- combined at the level of reduced cross sections $\sigma_{\text {red }}^{c \bar{c}}, \sigma_{\text {red }}^{b \bar{b}}$ $\sigma_{\text {red }}^{Q \bar{Q}}=\frac{\mathrm{d}^{2} \sigma^{Q \bar{Q}}}{\mathrm{~d} x_{\mathrm{Bj}} \mathrm{d} Q^{2}} \cdot \frac{x_{\mathrm{Bj}} Q^{4}}{2 \pi \alpha^{2}\left(1+(1-y)^{2}\right)}$ (full phase space) $(Q \bar{Q}$ stands either for $c \bar{c}$ or $b \bar{b})$

- combination accounts for correlation of systematic uncertainties, as well as correlation of $c$ and $b$ from same measurements

- $\Rightarrow$ significant improvement in precision via cross calibration of different measurement techniques and $c / b$

\section{Combined using HERAverager program}

[https://wiki-zeuthen.desy.de/HERAverager]

well established combination method used in:

- previous HERA charm combination [EPJ C73 (2013) 2311]

- HERAPDF2.0 [EPJ C75 (2015) 580]

- ATLAS papers [1603.09222, 1512.02192, 1606.01736, 1612.03016] 


\section{BACKUP. Input data}

[2] H. Abramowicz et al. [ZEUS Collaboration], "Measurement of $D^{ \pm}$Production in Deep Inelastic ep Scattering with the ZEUS detector at HERA", JHEP 05, (2013) 023 [arXiv: 1302.5058]

[3] H. Abramowicz et al. [ZEUS Collaboration], "Measurement of $D^{* \pm}$ Production in Deep Inelastic Scattering at HERA", JHEP 05, (2013) 097 [arXiv: 1303.6578]. Erratum-ibid JHEP 02, (2014) 106.

[4] H. Abramowicz et al. [ZEUS Collaboration], "Measurement of beauty and charm production in deep inelastic scattering at HERA and measurement of the beauty-quark mass", JHEP 09, (2014) 127 [arXiv: 1405.6915].

[5] H. Abramowicz et al. [ZEUS Collaboration], "Measurement of beauty production in deep inelastic scattering at HERA using decays into electrons", Eur. Phys. J. C71, (2011) 1573 [arXiv: 1 101.3692].

[6] H. Abramowicz et al. [ZEUS Collaboration], "Measurement of beauty production in DIS and F2bb extraction at ZEUS", Eur. Phys. J. C69, (2010) 347 [arXiv: 1005.3396]

[7] S. Chekanov et al. [ZEUS Collaboration], "Measurement of charm and beauty production in deep inelastic ep scattering from decays into muons at HERA", Eur. Phys. J. C65, (2010) 65 [arXiv:0904.3487].

[8] F. D. Aaron et al. [H1 Collaboration], "Measurement of the Charm and Beauty Structure Functions using the HI Vertex Detector at HERA". Eur. Phys. J. C65. (2010) 89 [arXiv:0907.2643]

[9] A. Aktas et al. [Hl Collaboration], "Production of D*+- Mesons with Dijets in Deep-Inelastic Scattering at HERA", Eur. Phys. J. C51, (2007) 271 [hep-ex/0701023]

[10] F. D. Aaron et al. [H1 Collaboration], "Measurement of $D^{* \pm}$ Meson Production and Determination of $F_{2}^{c c b a r}$ at low Q2 in Deep-Inelastic" Eur. Phys. J. C71, (2011) 1769 [arXiv: 1106.1028 ].

[11] F. D. Aaron et al. [H1 Collaboration], "Measurement of the D*+- Meson Production Cross Section and $\mathrm{F}(2)^{* *}(\mathrm{c}$ c-bar), at High Q**2, in ep Scattering at HERA", Phys. Lett. B686, (2010) 91 [arXiv:0911.3989].

[12] J. Breitweg et al. [ZEUS Collaboration], "Measurement of $\mathrm{D}^{*}+-$ production and the charm contribution to F2 in deep inelastic scattering at HERA", Eur. Phys. J. C12, (2000) 35 [hep-ex/9908012].

[13] S. Chekanov et al. [ZEUS Collaboration], "Measurement of $\mathrm{D}^{*}+-$ production in deep inelastic e+- p scattering at HERA", Phys. Rev. D69, (2004) 012004 [hep-ex/0308068].

[14] S. Chekanov et al. [ZEUS Collaboration], "Measurement of D+- and D0 production in deep inelastic scattering using a lifetime tag at HERA", Eur. Phys. J. C63, (2009) 171 [arXiv:0812.3775]. 


\section{BACKUP. Combination procedure}

- Take measured visible x-section $\sigma_{\text {vis }}$ and extrapolate to full phase space $\sigma_{\text {red }}$ using consistent NLO setup: $\sigma_{\text {red }}=\sigma_{\text {vis }} \frac{\sigma_{\text {red }}^{\mathrm{NLO}}}{\sigma_{\text {vis }}^{\mathrm{NLO}}}$ [HVQDIS]

- Combine $\sigma_{\text {red }}$ accounting for bin-to-bin correlations [HERAverager]

\section{NLO setup for extrapolation as in [DESY-12-172]}

- pole masses $m_{c}=1.5 \pm 0.15 \mathrm{GeV}, m_{b}=4.5 \pm 0.25 \mathrm{GeV}$ consistent with extracted from data: $m_{c}=1.43 \pm 0.04 \mathrm{GeV}, m_{b}=4.35 \pm 0.11 \mathrm{GeV}$ and consistent with PDG: $m_{c}=1.67 \pm 0.07 \mathrm{GeV}, m_{b}=4.78 \pm 0.06 \mathrm{GeV}$

- $\mu_{R}=\mu_{F}=\sqrt{Q^{2}+4 m_{Q}^{2}}$, varied simultaneously by factor 2

- $\alpha_{s}^{n_{f}=3}\left(M_{Z}\right)=0.105 \pm 0.002\left[\alpha_{s}^{n_{f}=5}\left(M_{Z}\right)=0.116 \pm 0.002\right]$

- HERAPDF1.0 FFNS, $n_{f}=3$, assign $2 \%$ uncor. unc. (checked vs HERAPDF2.0: see backup)

- $c$ fragmentation: Kartvelishvili frag. function parametrised as step function with $k_{T}$ kink (H1, ZEUS meas. [DESY-08-080, DESY-08-209])

- $b$ fragmentation: Peterson $\epsilon_{b}=0.0035 \pm 0.0020$ [NP B565 (2000) 245]

- charm fragmentation fractions [EPJ C76 (2016) 397]

- branching ratios PDG2016

- hadronisation uncertainties for data with jets in the final state 


\section{BACKUP. Combined data}

\section{BEAUTY}

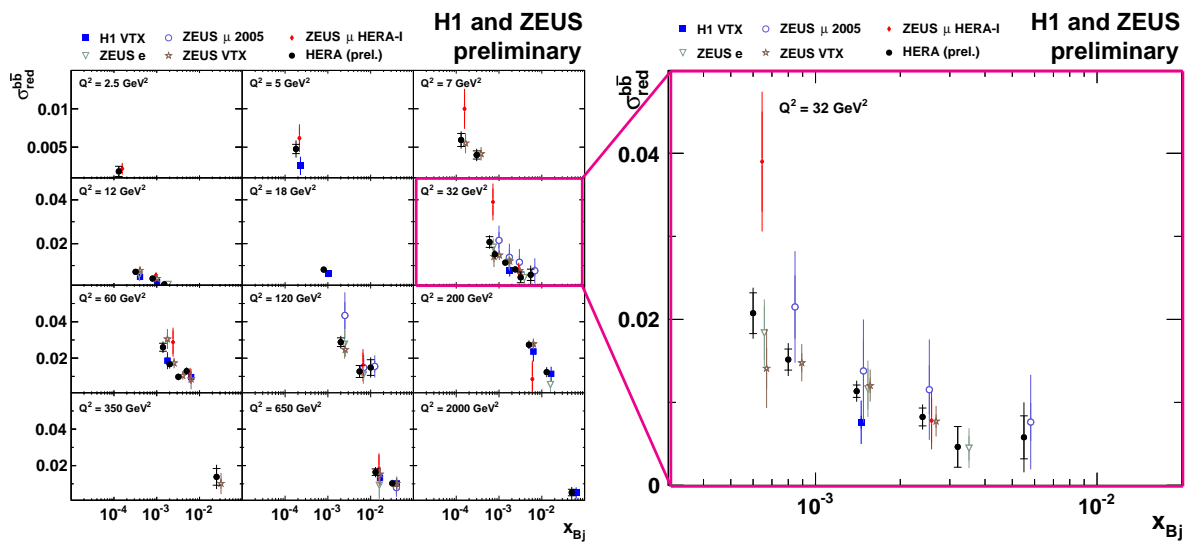

Significantly improved precision compared to input measurements 


\section{BACKUP. Data combination}

$\chi^{2}(\mathbf{m}, \mathbf{b})=\sum_{e=1}^{N_{e}} \sum_{i=1}^{N_{m}} \frac{\left(m_{i}-\sum_{j=1}^{N_{s}} \Gamma_{i}^{e, j} b^{e, j}-\mu_{i}^{e}\right)^{2}}{\sigma_{i}^{e 2}}+\sum_{j=1}^{N_{s}} b^{e, j^{2}}$

Minimised in iterative procedure 


\section{BACKUP. Theoretical predictions compared to data}

\section{Predictions calculated} with OPENQCDRAD interfaced in xFitter

www-zeuthen.desy.de/ alekhin/OPENQCDRAD ww. $x f i t t e r . o r g$

- input PDFs: HERAPDF2.0FF3A, ABM11, ABMP16, or fitted

- NLO or approx. NNLO as implemented in OPENQCDRAD

- $\mu_{f}=\mu_{r}=\sqrt{Q^{2}+4 m_{Q}^{2}}$, varied by factor 2 (dominant unc.)

- $m_{c}\left(m_{c}\right)=1.27 \pm 0.03 \mathrm{GeV}$, $m_{b}\left(m_{b}\right)=4.18 \pm 0.03 \mathrm{GeV}$ [PDG2016], or fitted

FFN scheme, $n_{f}=3$ : reliable in this kinematic range

\section{CHARM}

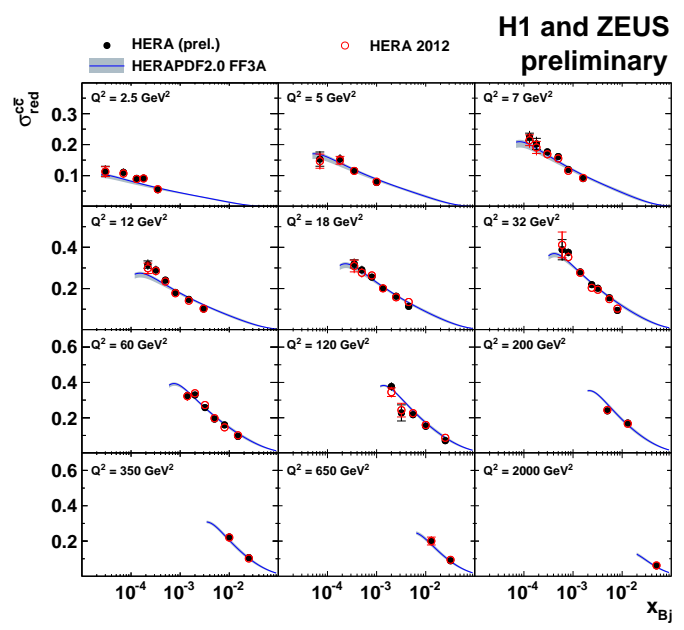

Overall reasonable description, some $x$ slope at low and medium $Q^{2}$ Same in previous H1ZEUS charm combination, but within larger unc. 


\section{BACKUP. Theoretical predictions compared to data}

\section{Predictions calculated with OPENQCDRAD interfaced in xFitter}

www-zeuthen.desy.de/ alekhin/OPENQCDRAD Ww. xfitter.org

- input PDFs: HERAPDF2.0FF3A, ABM11, ABMP16, or fitted

- NLO or approx. NNLO as implemented in OPENQCDRAD

- $\mu_{f}=\mu_{r}=\sqrt{Q^{2}+4 m_{Q}^{2}}$, varied by factor 2 (dominant unc.)

- $m_{c}\left(m_{c}\right)=1.27 \pm 0.03 \mathrm{GeV}$, $m_{b}\left(m_{b}\right)=4.18 \pm 0.03 \mathrm{GeV}$ [PDG2016], or fitted

FFN scheme, $n_{f}=3$ : reliable in this kinematic range

\section{BEAUTY}

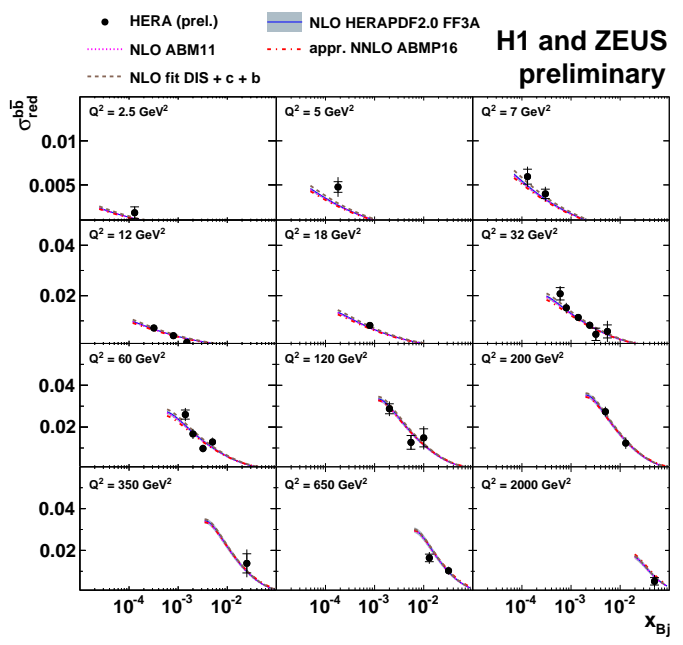

Overall good description within data uncertainties 


\section{BACKUP. Theoretical predictions compared to data}

Predictions calculated with OPENQCDRAD interfaced in $x$ Fitter

www-zeuthen.desy.de/ alekhin/OPENQCDRAD ww. xfitter.org

- input PDFs: HERAPDF2.0FF3A, ABM11, ABMP16, or fitted

- NLO or approx. NNLO as implemented in OPENQCDRAD

- $\mu_{f}=\mu_{r}=\sqrt{Q^{2}+4 m_{Q}^{2}}$, varied by factor 2 (dominant unc.)

- $m_{c}\left(m_{c}\right)=1.27 \pm 0.03 \mathrm{GeV}$, $m_{b}\left(m_{b}\right)=4.18 \pm 0.03 \mathrm{GeV}$ [PDG2016], or fitted

FFN scheme, $n_{f}=3$ : reliable in this kinematic range

\section{BEAUTY}

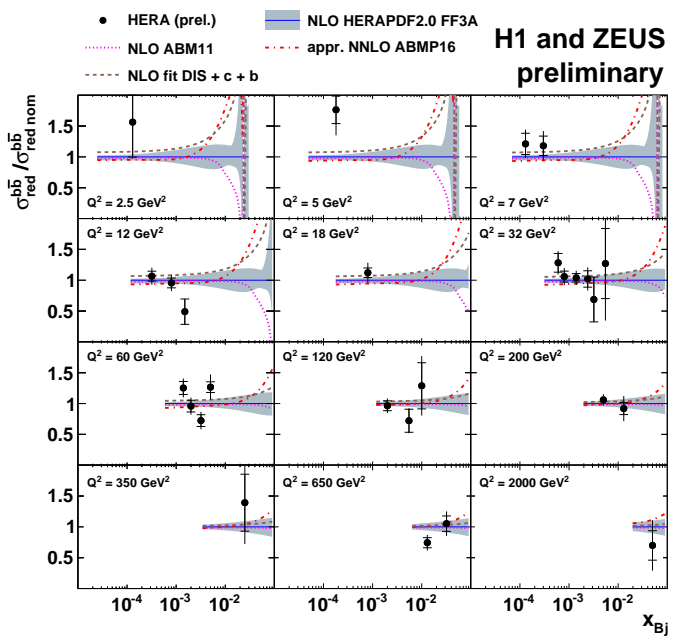

Overall good description within data uncertainties Small sensitivity to PDFs and higher order corrections 


\section{BACKUP. Theoretical predictions compared to data}

\begin{tabular}{|l|l|l|l|}
\hline Dataset & PDF & $\chi^{2}$ & $\chi^{2}$ with PDF unc. \\
\hline \hline \multirow{2}{*}{ HERA 2012 $c$ [1] } & HERAPDF20_NLO_FF3A_EIG & 59 & 59 \\
abm11_3n_nlo & 62 & 62 \\
\hline \hline \multirow{2}{*}{ New combined $c$} & HERAPDF20_NLO_FF3A_EIG & 86 & 63 \\
\hline \multirow{2}{*}{ (dof = 52) } & abm11_3n_nlo & 92 & 85 \\
\hline \hline \multirow{2}{*}{ ZEUS VTX $b$ [4] } & ABMP16_3_nnlo & 101 & 91 \\
(dof = 17) & abm11_3n_nlo & ABMP16_3_nnlo & 14 \\
\hline \hline \multirow{2}{*}{ New combined $b$} & HERAPDF20_NLO_FF3A_EIG & 33 & 13 \\
$($ dof $=$ 27) & abm11_3n_nlo & 34 & 14 \\
\hline
\end{tabular}

[1] previous HERA charm combination EPJ C73 (2013) 2311

[4] ZEUS $b$ lifetime tagging measurement JHEP09 (2014) 127

(most precise individual public data sets for $c$ and $b$ from HERA to date)

\section{Quantitatively confirms observed findings:}

- larger tension for new charm data owing to reduced uncertainties

- appr. NNLO does not improve data description compared to NLO

- overall small sensitivity to input PDFs 


\section{BACKUP. QCD analysis settings}

Similar to HERAPDF2.0 FF, using running $\mathrm{HQ}$ mass definition:

- xFitter-1.2.0

- Input data:

- HERA $e^{ \pm} p$ inclusive data, $Q_{\min }^{2}>3.5 \mathrm{GeV}^{2}$ [1506.06042]

- new HERA $c$ and $b$ combined

- FFNS $n_{f}=3$ ('FF ABM RUNM'), $\left(\alpha_{s}\left(F_{L}\right)=\alpha_{s}\left(F_{2}\right)\right.$ )

- $\alpha_{s}^{n_{f}=3}\left(M_{Z}\right)=0.106$

- free $m_{c}\left(m_{c}\right), m_{b}\left(m_{b}\right)$, or PDG $m_{c}\left(m_{c}\right)=1.27 \mathrm{GeV}, m_{c}\left(m_{c}\right)=4.18 \mathrm{GeV}$

- DGLAP NLO [QCDNUM]

- PDF parametrisation: 14 p HERAPDF at $\mu_{f 0}^{2}=1.9 \mathrm{GeV}^{2}, f_{s}=0.4$ :

$$
\begin{aligned}
& x g(x)=A_{g} x^{B g}(1-x)^{C g}-A_{g}^{\prime} x^{B_{g}^{\prime}}(1-x)^{C_{g}^{\prime}} \\
& x u_{v}(x)=A_{u_{v}} x^{B u_{v}}(1-x)^{C_{u_{v}}}\left(1+E_{u_{v}} x^{2}\right) \\
& x d_{v}(x)=A_{d_{v}} x^{B} d_{v}(1-x)^{C_{d_{v}}} \\
& x \bar{U}(x)=A_{\bar{U}} x^{B} \bar{U}(1-x)^{C} \bar{U}\left(1+D_{\bar{U}} x\right) \\
& x \bar{D}(x)=A_{\bar{D}} x^{B} \bar{D}(1-x)^{C_{\bar{D}}}
\end{aligned}
$$$$
\mid \begin{aligned}
& \text { Additional constrains: } \\
& A_{\bar{U}}=A_{\bar{D}}\left(1-f_{s}\right), B_{\bar{U}}=B_{\bar{D}}, C_{g}^{\prime}=25 \\
& \int_{0}^{1}\left[\sum_{i}\left(q_{i}(x)+\bar{q}_{i}(x)\right)+g(x)\right] x d x=1 \\
& \int_{0}^{1}[u(x)-\bar{u}(x)] d x=2, \\
& \int_{0}^{1}[d(x)-\bar{d}(x)] d x=1
\end{aligned}
$$

- fit $\left(\Delta \chi^{2}=1\right)$, model (scales, $\left.\alpha_{s}, f_{s}, Q_{\min }^{2}\right)$ and par. $\left(\mu_{f 0}, E_{u_{v}}=0\right)$ unc. 


\section{BACKUP. Discussion of HQ mass extraction}

$$
\begin{aligned}
& \boldsymbol{m}_{\boldsymbol{c}}\left(\boldsymbol{m}_{\boldsymbol{c}}\right)=1290_{-41}^{+46}(\mathrm{fit})_{-14}^{+62}(\bmod )_{-31}^{+7}(\mathrm{par}) \mathrm{MeV}
\end{aligned}
$$

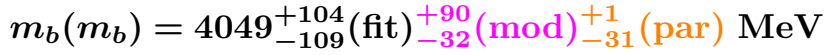

Results have sizable model and parametrisation uncertainty:

- model uncertainties dominated by scale variations

- parametrisation uncertainties dominated by reduced $13 p$ form: closely related to inclusive HERA data in the fit

Using inclusive HERA data only:

$m_{c}\left(m_{c}\right)=1798_{-134}^{+144}$ (fit) $\mathrm{MeV}$

$m_{b}\left(m_{b}\right)=8450_{-1810}^{+2280}$ (fit) $\mathrm{MeV}$

No full uncertainty evaluation, but observed large sensitivity to PDF parametrisation $(\rightarrow 13 p)$ :

$$
\begin{aligned}
& m_{c}\left(m_{c}\right)=1798 \rightarrow 1450 \mathrm{MeV}, \\
& m_{b}\left(m_{b}\right)=8450 \rightarrow 3995 \mathrm{MeV}
\end{aligned}
$$

$$
\begin{aligned}
& x g(x)=A_{g} x^{B}(1-x)^{C_{g}}-A_{g}^{\prime} x^{B_{g}^{\prime}(1-x)^{C_{g}^{\prime}}} \\
& x u_{v}(x)=A_{u_{v}} x^{B} u_{v}(1-x)^{C_{u_{v}}\left(1+E_{u_{v}} x^{2}\right)} \\
& x d_{v}(x)=A_{d_{v}} x^{B} d_{v}(1-x)^{C_{d_{v}}} \\
& x \bar{U}(x)=A_{\bar{U}} x^{B} \bar{U}(1-x)^{C_{\bar{U}}}\left(1+D_{\bar{U}} x\right) \\
& x \bar{D}(x)=A_{\bar{D}} x^{B} \bar{D}(1-x)^{C} \bar{D}
\end{aligned}
$$

$$
\text { 13p: } E_{u_{v}}=0
$$

$\Rightarrow$ inclusive HERA data alone cannot constrain HQ masses

$\Rightarrow$ interplay of PDFs and HQ masses needs carefull treatment 


\section{BACKUP. $m_{c}\left(m_{c}\right)$ extraction in FFNS and VFNS}

JHEP 1608 (2016) 050

\begin{tabular}{lcc}
\hline variation & FONLL-C & FFN \\
\hline \hline central & $1.335 \pm 0.043$ & $1.318 \pm 0.054$ \\
\hline \hline$Q_{0}^{2}=1.5$ & $1.354[+0.019]$ & $1.329[+0.011]$ \\
\hline$D_{u v}$ non-zero & $1.340[+0.005]$ & $1.308[-0.010]$ \\
\hline \hline$f_{s}=0.3$ & $1.338[+0.003]$ & $1.320[+0.002]$ \\
$f_{s}=0.5$ & $1.332[-0.003]$ & $1.315[-0.003]$ \\
\hline$m_{b}\left(m_{b}\right)=3.93 \mathrm{GeV}$ & $1.330[-0.005]$ & $1.312[-0.006]$ \\
$m_{b}\left(m_{b}\right)=4.43 \mathrm{GeV}$ & $1.343[+0.008]$ & $1.324[+0.006]$ \\
\hline$\alpha_{s}\left(M_{Z}\right)=0.1165$ & $1.342[+0.007]$ & $1.332[+0.014]$ \\
$\alpha_{s}\left(M_{Z}\right)=0.1195$ & $1.329[-0.006]$ & $1.300[-0.018]$ \\
\hline \hline$\mu_{F}^{2}=\mu_{R}^{2}=2 \cdot Q^{2}$ & $1.347[+0.012]$ & $1.314[-0.004]$ \\
$\mu_{F}^{2}=\mu_{R}^{2}=Q^{2} / 2$ & $1.361[+0.026]$ & $1.363[+0.045]$ \\
\hline FONLL Damping power $=1$ & $1.352[+0.017]$ & - \\
FONLL Damping power $=4$ & $1.327[-0.008]$ & - \\
\hline
\end{tabular}

\section{A determination of $m_{c}\left(m_{c}\right)$ from HERA data using a matched heavy-flavor scheme}

- consistent results obtained in FFNS and FONLL, with somewhat different decomposition of uncertainties

- $\Rightarrow$ VFNS can be used for $\overline{\mathrm{MS}}$ mass extraction, if all uncertainties from extra parameters are considered 